\title{
A HARMONIC ANALYSIS VIEW ON NEUROSCIENCE IMAGING
}

\author{
PAUL HERNANDEZ-HERRERA, DAVID JIMÉNEZ, IOANNIS A. KAKADIARIS, \\ ANDREAS KOUTSOGIANNIS, DEMETRIO LABATE, FERNANDA LAEZZA, MANOS PAPADAKIS
}

\begin{abstract}
After highlighting some of the current trends in neuroscience imaging, this work studies the approximation errors due to varying directional aliasing, arising when $2 \mathrm{D}$ or 3D-images are subjected to the action of orthogonal transformations. Such errors are common in 3D-images of neurons acquired by confocal microscopes. We also present an algorithm for the construction of synthetic data (computational phantoms) for the validation of algorithms for the morphological reconstruction of neurons. Our approach delivers synthetic data that have a very high degree of fidelity with respect to their ground-truth specifications.
\end{abstract}

\section{OVERTURE}

What is the substance of knowledge and memory? These fundamental questions have been at the center of philosophical debate for over three millenia, but only during the last fifty years our understanding of these essential human cognitive functions is finally becoming concrete. The quest for answers takes us back to the philosopher Plato (424/423 BC $-348 / 347$ BC) who, in the dialogue "Theaetetus", written circa 360 B.C. when Athens' glory was in decline amidst the Peloponnesian war, attempts to define knowledge from a philosophical viewpoint. In the dialogue, Euclid (not the famous geometer from Alexandria) recounts a discussion between Socrates and Theaetetus aiming to discover the nature of knowledge. Around the middle of their conversation Socrates refers to knowledge as being a series of 'engrams', impressions on the 'wax of the soul':

SOCRATES: And the origin of truth and error is as follows: When the wax in the soul of any one is deep and abundant, and smooth and perfectly tempered, then the impressions which pass through the senses and sink into the heart of the soul, as Homer says in a para-

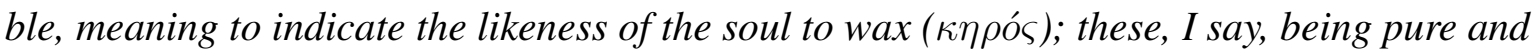
clear, and having a sufficient depth of wax, are also lasting, and minds, such as these, easily learn and easily retain, and are not liable to confusion, but have true thoughts, for they have plenty of room, and having clear impressions of things, as we term them, quickly distribute

Key words and phrases. Synthetic tubular data, synthetic dendrites, directional aliasing, approximation error, dendritic arbor segmentation, confocal microscopy.

P. Hernandez-Herrera, and I. A. Kakadiaris are with the Computational Biomedicine Lab, Department of Computer Science, University of Houston, Houston, Texas 77204, USA; D. Jiménez, D. Labate, and M. Papadakis are with the Department of Mathematics, University of Houston, Houston, Texas 77204-3008, USA; A. Koutsogiannis is with the University of Athens, Greece, Department of Mathematics, GR-15784 Zografou, Greece; F. Laezza is with the Department of Pharmacology and Toxicology, University of Texas Medical Branch, Galveston, Texas 77555-1031, USA. Email for all correspondence: mpapadak@math.uh.edu. 
them into their proper places on the block. And such men are called wise. Do you agree?

\section{THEAETETUS : Entirely.}

SOCRATES: But when the heart of any one is shaggy a quality which the all-wise poet commends, or muddy and of impure wax, or very soft, or very hard, then there is a corresponding defect in the mind the soft are good at learning, but apt to forget; and the hard are the reverse; the shaggy and rugged and gritty, or those who have an admixture of earth or dung in their composition, have the impressions indistinct, as also the hard, for there is no depth in them; and the soft too are indistinct, for their impressions are easily confused and effaced. Yet greater is the indistinctness when they are all jostled together in a little soul, which has no room. These are the natures which have false opinion; for when they see or hear or think of anything, they are slow in assigning the right objects to the right impressions in their stupidity they confuse them, and are apt to see and hear and think amiss and such men are said to be deceived in their knowledge of objects, and ignorant.

\section{THEAETETUS: No man, Socrates, can say anything truer than that 1 .}

With the 'wax of the soul' theory, Greek philosophers anticipated the impressively modern concept of the human brain and its plastic neuronal network connections as the site of memory engrams formation and knowledge retention [24, 23]. Despite the impressive advances of modern science, however, our journey towards the comprehension of the physical nature of the 'wax of the soul' and of the memory engrams is still at the 'end of the beginning'. We are optimistic that through interdisciplinary, collective scientific efforts this mystery will be finally unlocked.

1.1. Outline. This article is organized as follows. In Section 2, we provide a brief historical overview of neuroscience and describe the challenges and opportunities opened up by the recent advances in microscopy. In particular, we discuss the significance of developing computational tools for the morphological reconstruction of neurons. Next, in Section 3, we give an overview of the algorithms currently available for the segmentation and morphological reconstruction of neurons, including a brief account of ORION, a suite of algorithms and software developed by some of the authors of this paper which provides semi-automatic segmentation and morphological reconstruction of dendritic arbors in neurons. In Section 4 we examine the aliasing errors arising when images are subjected to the action of orthogonal transformations. Such errors are common in 3D-images of neurons acquired by confocal microscopes. The action of those orthogonal transformations modifies the frequency content of images during the conversion of an image from analog to digital as the high-frequency content may be enhanced or attenuated solely due to the action of an orthogonal transformation. We also provide error estimates and sufficient conditions for the sampling kernels guaranteeing that these reconstruction error estimates are not affected by the action of a group of orthogonal transformations. Finally, in Section 5 , we use the results of Section 4 to develop an algorithm for the construction of highly accurate phantoms of tubular 3D-structures which

\footnotetext{
${ }^{1}$ Translated by Benjamin Jowett [29]
} 
are useful to model realistic phantoms of dendritic arbors of arbitrary topological complexity and can be used for the benchmarking of segmentation and tracing algorithms.

\section{THE SAGA}

2.1. Historical background. The concept of the neuron as the primary structural unit of the central nervous system was introduced as early as the 19th century by the ground-breaking studies of Camillo Golgi (1843-1926) and Ramón y Cajal (1852-1934). Utilizing an ingenious tissue staining technique developed by Golgi, Ramón y Cajal provided the earliest evidence of the neuron as the primary discrete unit of the central nervous system, and defined its micro-anatomy using light microscopy. By examining the structure of thousands of neurons in every region of the brain, Ramón y Cajal discovered the universal structure of neurons consisting of a cell body (also called the soma), dendrites, and an axon. With impressive accuracy, he also postulated that dendrites, which are multiple branching structures that arise from the cell body, and the axon, a single elongated cellular protrusion stemming from the cell body, retain different functions and mediate specialized connections between neurons. In following studies in 1933, Ramón y Cajal conjectured that neuronal spines, which are the protuberances appearing along the dendrites (similar to rose thorns hence called espinas), are the points where these specialized connections through which the axon of one neuron contacts a neighboring neuron are established. Remarkably, he postulated that spines are a manifestation of the economy of nature: they increase the surface of a dendrite enabling stronger connections between neural cells via the dendrite-axon route [77, pages 3,101]. These connections are now referred to as synapses ${ }^{2}$ and constitute the fundamental structures that permit a neuron to transmit electrochemical signals to another neighboring cell (usually to another neuron). With these fundamental studies, Ramón y Cajal laid the foundations of modern neuroscience. While neuroanatomy studies were flourishing, in the 1930's, Curtis, Cole, Hodgkin and Huxley, four neurophysiologists, were investigating the electrical properties of the axon of the Atlantic giant squid, a large and easily accessible tissue preparation, and provided the first recordings of the action potential, a form of regenerative electrical waveform that propagates down the axon [15, 27]. With the use of the voltage clamp technique, Hodgkin and Huxley discovered that the action potential arises from sequential changes in the cell membrane permeability to $\mathrm{Na}^{+}$and $\mathrm{K}^{+}$ions, and developed the first mathematical model of the action potential propagation using non-linear differential equations. For the first time in history, these experiments revealed the basis of electric function in neurons. Later studies in the 1950's by Fatt, Katz and del Castillo [33] established that, by propagating down the axon, the action potential mediates synaptic transmission. Once it reaches the presynaptic bouton (the large ending of the axon), the action potential is decoded into a chemical signal through the release of discrete quanta of neurotransmitter molecules which eventually reach the postsynaptic side of the synapse (spine) and bind to specific membrane ion channels, called receptors. Upon binding to the neurotransmitter, receptors change conformation allowing specific ions to permeate into the postsynaptic cell and generate an electric charge called the excitatory postsynaptic potential (EPSP). If this

\footnotetext{
${ }^{2}$ from the Greek prefix ' $\sigma v \nu-$ ' and the root of the verb ' $\alpha \pi \tau o \mu \alpha \iota$ ', to touch; by adding the prexif the verb $\sigma v \nu \alpha \dot{\alpha} \tau \omega \omega$ means to clasp together but in ancient and in modern Greek it means 'to form an accord or to establish a formal relationship'
} 
electric charge exceeds a certain threshold, the EPSP elicits an action potential in the postsynaptic cell (the receiving cell). It is through this sequence of electrochemical chain-reactions that the information is transmitted and stored in the brain through a connectome of neuronal networks. Although these basic concepts of neurophysiology are very well-established, the explosive development of ultra-sophisticated, unprecedented resolution imaging technologies in modern times have revealed new fascinating aspects of synaptic transmission and specifically have highlighted the critical role of synaptic spines as the main integrators of neuronal information.

2.2. Modern neuroscience. The emergence of fluorescence-based technologies and the development of sophisticated fluorescence microscopes, such as confocal and multi-photon, facilitate the acquisition of high-resolution fluorescent images of dendrites and spines both in vitro and in vivo and allows the monitoring of their dynamic structural changes in realtime. It is now well documented that spines can grow or disappear in response to rapid and local changes in synaptic transmission (spine plasticity) or to more global and prolonged effects induced by network activity (neuronal homeostasis). These dynamic morphological changes of spines, associated with plasticity and homeostasis, are considered to be the structure-function link in the heart of learning and memory formation and are associated with different behavioral states or chronic neuropathologies [64, 63, 24, 79, 23, 78, 77]. It is through structure-function changes of synaptic spines throughout neuron networks that we retain what we have learned, we respond to external stimuli, and eventually we adapt to the surrounding environment. With no doubts, the ability to accurately capture the morphological information of dendrites and spines and track their dynamic changes will rapidly translate into a better understanding of brain function. Towards futuristic applications this improved knowledge of cellular and sub-cellular neuronal morphologies could be included in electrophysiological computer simulations, so that quantitative and qualitative effects of dendritic and spine structure under stimulation can be extensively characterized. With the current computational capabilities, these models can be implemented into supercomputers to allow the generation of virtual neurons which retain all anatomical and functional characteristics of their real counterparts. When modeled neurons are organized into complex structures under appropriate rules governing their anatomical and functional connectivity, in principle, entire portions of the nervous system would be simulated into realistic neural networks, leading to what G. Ascoli phrased as: 'A detailed computer model of a virtual brain that was truly equivalent to the biological structure' and 'could in principle allow scientists to carry out experiments that could not be performed on real nervous systems because of physical constraints' [2].

While the technological advances in fluorescence-based microscopy have opened up exciting avenues of investigation in neuroscience and have set high-standard goals to modern neurophysiology, this area of research has also raised a number of computational, algorithmic and mathematical challenges involving the acquisition and modeling of high resolution data acquired through confocal microscopy, the preprocessing of the data (which are typically affected by blurring and Poisson noise), and the morphological reconstruction of dendritic structures and spines. Capturing and accurately modeling the morphological transitions of spines and dendrites in response to various functional states of a neuron will bring us a step closer to identify the physical nature of the 'wax of the soul' anticipated by Plato and 
to unravel how memory traces or engrams are formed, retained are translated into human cognitive functions [24, 23].

\section{IMAGING NEURONS}

3.1. The state of the art. As indicated by the observations in the section above, the ability to produce accurate morphological reconstruction of dendritic arbors and spines in neurons is of fundamental importance to the goal of generating a virtual neuron. During the last ten years, a flurry of activity was aimed at the development of automatic or semi-automatic computational tools for delivering morphological reconstructions of neurons and there are currently several academic and a few commercial and freeware imaging suites available. All of these algorithms depict branching and terminal points, diameters of dendritic branches and the soma and output the results in 3D-visualizations. Their performance varies and depends on the level of training per data set, the noise that affects the data and on the level of manual intervention required. These reconstructions rely on a tracing of the dendritic arbor which has been a hot research topic, the least, in the last ten years e.g. [31, 40, 42, 62, 26, 43, 46, 47, 37, 58, 73, 51, 21, 48, 61, 44, 53, 74, 1, 76, 41, 52, 22]. More recently, significant work on the tracing and morphological reconstruction of dendritic arbors emerged as a result of the DIADEM competition [8, 44]. Although, all of them are designed to capture the 3D-structure of the dendritic arbor with sufficient accuracy, they usually miss the spatially localized detail of the surface of the dendritic branches and in particular they ignore spines [61] as the common goal of all of the dendrite-tracing methods is to detect the centerline of dendritic branches. This naturally and reasonably becomes a new system of coordinates for navigating the dendrite. Although several of the dendrite tracing algorithms estimate the dendritic diameter locally, their estimations cannot capture the localized details of the dendritic surface with the exception of [59, 38, 39, 32, 57, 58, 31, 60] which generates a probabilistic segmentation of the volume of the dendritic arbor; thus the likelihood of the association of a voxel to the dendritic surface is obtained.

The existing spine detection capabilities of current 3D algorithms build upon the type of centerline tracing we previously described. Using the detected centerline for navigation within the dendrite they typically apply the Rayburst detection algorithm [75, 54, 66]. Several other methods rely on detecting spines on 2D-maximum intensity projections but those methods frequently miss significant spines and the many of the weaker ones as they are obscured by the projection of the higher intensity parts of the dendritic volume onto them [4, 14]. In particular, Fan et. al use maximum intensity projections for in-vivo spine detection and analysis [20]. There is very limited work on in-vivo spine detection primarily because of the necessity to use tracking algorithms when 2D-image analysis methods are employed. There are also pseudo-3D approaches in the sense that spines are detected on each scanning plane and then the results of the detection are fused to create a 3D-image stack [19, 80, 13, 12, 3, 81, 50]. Classification of spines according to their types, estimation of volume and of head diameter is mainly being done with the Rayburst algorithm [18]-often applied in 2D-only [36, 56]- which counts voxels whose intensity exceeds an operator-chosen threshold on certain directions. However, two are the main problems in all of these approaches: 
a. The use of intensity thresholds applied on the original image in order to detect the surface of spines. Since Poisson noise corrupts images, intensity thresholds are increasingly unreliable as the concentration of the fluorophores decreases. This is often the case in undeveloped or thin spines but more importantly it affects spine necks resulting into detached spines which are harder to distinguish from leaking fluorophores or plain noise spikes.

b. Constraints of technical nature as well as the need to decrease image acquisition duration lead to the use of anisotropic voxels, typically of aspect ratio 1:1:3 to 1:1:4. Although images are corrected to account for blurring introduced by the microscope, dendritic volumes are reconstructed with voxels of these aspect ratios. This implies that objects such as spines which are oriented in an arbitrary way in 3D and have a diameter of 10-13 voxels with their neck being less than 2-3 voxels thick at the highest resolution will not be properly classified according to their shape as their shape is distorted by this anisotropic sampling grid. A partial heuristic remedy utilizing the Rayburst algorithm is proposed in [18] to mitigate this problem but it can only have limited success since the data are severely undersampled at off the $x y$-plane orientations.

\subsection{Online Reconstruction and functional Imaging of Neurons (ORION). ORION [72}

59, 38, 39, 32, 57, 58, 31, 60] is a suite of algorithms and integrated software that can be used for the morphological reconstruction of dendritic arbors from 3D-images obtained by multiphoton or confocal microscopes. ORION can identify dendritic centerlines, their branching and terminal points and estimates the diameter of branches at every centerline point; however, it does not identify or classify spines and 3D-visualizations of the morphological reconstructions of dendritic arbors do not include spines [11]. ORION segmentation of the dendritic arbor is based on extracting the eigenvalues of the Hessian of an ensemble of low-pass Gaussian filtered outputs of the original 3D-volume and by learning how these eigenvalues depend on a tubular model estimated from the data. The segmented volume results from a probability 3D-map conditioned on the learned model. Dendritic centerlines and branching points representing the unique solution of a certain optimization problem. ORION has been successfully tested on synthetic data, on real data where the system outperformed experts and on several DIADEM competition image sets. Notice however that, since ORION is designed to work primarily on dendritic arbors that are acyclic connected graphs, it cannot be applied to some of the DIADEM data sets. Figure 1 illustrates an application of ORION.

\section{Approximations under the ACtion of a Group of ORThOgOnAL TRANSFORMATIONS}

Since all images have compact support in the space domain $\mathbb{R}^{d}(d=2$ or $d=3)$, their conversion from analogue to digital form occurring during acquisition requires truncating the image in the frequency domain. Typically, this process is modeled by convolving the given image, say $f \in L^{2}\left(\mathbb{R}^{d}\right)$, with a kernel function $\phi_{a}$, referred to as the analysis kernel. Hence, if $0<\varepsilon<1$ is a preselected constant representing the level of the desired relative error, there is a compact subset of the frequency domain, say $\Omega$, such that $\int_{\Omega^{c}}|\widehat{f}(\xi)|^{2} \mathrm{~d} \xi<\varepsilon\|\widehat{f}\|_{2}^{2}$. The set $\Omega$ is called the essential bandwidth of $f$. With no loss of generality we assume 


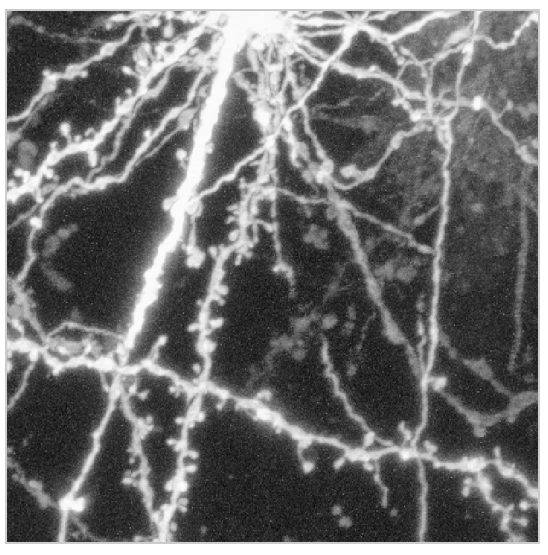

(a)

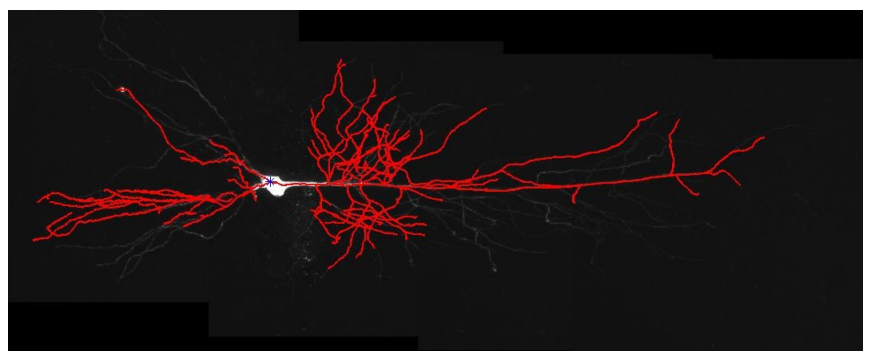

(b)

FIGURE 1. Left: MIP of the logarithm of a raw image of CA1 hyppocampal pyramidal neuron labelled with DiI fluorescent dye demonstrating the nature of the noise in voxels next to the dendritic branches. Right: Morphological reconstruction of a pyramidal neuron with ORION. The segmented dendrite's voxels are color-coded red. Note the absence of spines

$\Omega \subseteq \mathbb{T}^{d}=[-1 / 2,1 / 2]^{d}$. In particular, we define

$$
\mathcal{B}_{\Omega}^{\varepsilon}:=\left\{f \in L^{2}\left(\mathbb{R}^{d}\right): \hat{f} \in W^{1,2}\left(\mathbb{R}^{d}\right) \text { and } \int_{\Omega^{c}}|\widehat{f}(\xi)| \mathrm{d} \xi<\varepsilon\|\widehat{f}\|_{1}\right\},
$$

where $W^{1,2}\left(\mathbb{R}^{d}\right)$ is the Sobolev space containing all functions $h$ whose distributional partial derivatives up to second-order are contained in $L^{1}\left(\mathbb{R}^{d}\right)$. We can view $\mathcal{B}_{\Omega}^{\varepsilon}$ as a family of functions that are almost bandlimited, as for a function bandlimited in $\Omega$ one would have $\int_{\Omega^{c}}|\widehat{f}(\xi)| \mathrm{d} \xi=0$. This observation motivates us to generalize classical sampling theory approaches in the spirit of [5]. Specifically, we adopt an oversampling approach implementing the digitization of the input image and its reconstruction from its samples. To this end we use two kernels, the analysis kernel $\phi_{a}$ and the synthesis kernel $\phi_{s}$. Two compact sets are associated with this pair of kernels, $B_{a}$ and $B_{s}$. We assume $\Omega \subset B_{a}^{\circ}, B_{a} \subset B_{s}^{\circ}$, and $B_{s} \subset\left(\mathbb{T}^{d}\right)^{\circ}$, where the superscript $\circ$ indicates the interior of a set, and

(1) $\widehat{\phi}_{a}, \widehat{\phi}_{s} \subset C^{\infty}\left(\mathbb{R}^{d}\right) \cap L^{1}\left(\mathbb{R}^{d}\right)$,

(2) All partials derivatives of $\widehat{\phi}_{a}$ and $\widehat{\phi}_{s}$ up to second order are bounded,

(3) $\left|\widehat{\phi}_{a}(\xi)\right| \leq 1+\varepsilon,\left|\widehat{\phi}_{s}(\xi)\right| \leq 1+\varepsilon$ for all $\xi \in \mathbb{R}^{d}$,

(4) $\left|\widehat{\phi}_{a}(\xi)-1\right| \leq \varepsilon$ if $\xi \in B_{a},\left|\widehat{\phi}_{a}(\xi)\right| \leq \varepsilon$ if $\xi \notin B_{s}$,

(5) $\left|\widehat{\phi}_{s}(\xi)-1\right| \leq \varepsilon$ if $\xi \in B_{s},\left|\widehat{\phi}_{s}(\xi)\right| \leq \varepsilon$ if $\xi \notin \mathbb{T}^{d}$,

(6) $\int_{B_{a}^{c}}\left|\widehat{\phi}_{a}(\xi)\right| \mathrm{d} \xi<\varepsilon$ and $\int_{\left(\mathbb{T}^{d}\right)^{c}}\left|\widehat{\phi}_{s}(\xi)\right| \mathrm{d} \xi<\varepsilon$

(7) there exists $C>0$ such that $\sum_{k \in \mathbb{Z}^{d}}\left|\hat{\phi}_{a}(\xi+k)\right|^{2} \leq C$ and $\sum_{k \in \mathbb{Z}^{d}}\left|\hat{\phi}_{s}(\xi+k)\right|^{2} \leq C$ for a.e. $\xi \in \mathbb{R}^{d}$. 
With these conditions in mind we define

$$
\tilde{f}:=\sum_{n \in \mathbb{Z}^{d}}\left\langle f, T_{n} \phi_{a}\right\rangle T_{n} \phi_{s}
$$

where the right-hand side of (2) converges with respect to the $L^{2}$-norm due to Property (7) above. Notice that Property (1) above guarantees that both kernels have good spatial localization. Properties (3) and (4) indicate that $B_{a}$ and $B_{s}$ are the pass-bands of $\phi_{a}$ and $\phi_{s}$ respectively, while their stop-bands are both contained in $\mathbb{T}^{d}$. The digitization process gives the sequence $\left\{\left\langle f, T_{n} \phi_{a}\right\rangle: n \in \mathbb{Z}^{d}\right\}$, while the inversion of this process, the reconstruction of original analog image from its samples $\left\langle f, T_{n} \phi_{a}\right\rangle$ is referred to as the digital to analog conversion. Typically, in imaging we only use the first part the analog to digital conversion, while the reverse process has only theoretical value. In general, $f \neq \tilde{f}$. It is one of our goals to estimate $\|f-\tilde{f}\|$, called the reconstruction error, with respect to different meaningful norms. In the applications presented in this paper, the $L^{\infty}$-norm is the proper norm because it guarantees the uniform fidelity of the reconstruction of $f$ throughout the spatial domain. In practice though, it is impossible to keep an infinite number of the samples $\left\{\left\langle f, T_{n} \phi_{a}\right\rangle\right\}_{n \in \mathbb{Z}^{d}}$. Therefore, it becomes necessary to make a choice of a finite set $\Lambda \subset \mathbb{Z}^{d}$ such that the only values kept belong to $\left\{\left\langle f, T_{n} \phi_{a}\right\rangle\right\}_{\Lambda}$. Hence,

$$
f_{\Lambda}=\sum_{n \in \Lambda}\left\langle f, T_{n} \phi_{a}\right\rangle T_{n} \phi_{s}
$$

gives an approximation of the original input signal or image $f$. Specializing to images, their finite extend and the limitations of the acquisition devices prescribe a certain size of voxels/pixels. This mathematically amounts to prescribing a certain essential bandwidth which has the form of a parallelepiped in $\mathbb{R}^{d}(d=2,3)$ and $\Lambda=\prod_{s=1}^{d}\left[-N_{s}, N_{s}\right]$, where all $N_{s}$ are integers. So it is important to study the overall approximation error $\left\|f-f_{\Lambda}\right\|$, with respect to various norms. In this paper we are interested in the approximation error with respect to the $L^{\infty}$-norm and in particular, we propose how to control this error when $f$ varies, due to the action of a group of orthogonal transformations defined on $\mathbb{R}^{d}$. Specifically, given a group $G$ of orthogonal transformations acting on $\mathbb{R}^{d}$, e.g. $G=S O(d)$, we want to be able to find suitable kernels $\phi_{a}$ and $\phi_{s}$ so that iffor a choice of $\Lambda \subseteq \mathbb{Z}^{d}$ (e.g. $\Lambda=\prod_{s=1}^{d}\left[-N_{s}, N_{s}\right]$ ) the error $\left\|f-f_{\Lambda}\right\|_{\infty}<\epsilon$, that is $\left\|f-f_{\Lambda}\right\|_{\infty}$ is small enough, then

$$
\sup _{M \in G}\left\|\rho(M) f-(\rho(M) f)_{\Lambda}\right\|_{\infty} \leq \epsilon,
$$

where $\rho(M) f(x)=f(M x), x \in \mathbb{R}^{d}$.

Since,

$$
\left\|\rho(M) f-(\rho(M) f)_{\Lambda}\right\|_{\infty} \leq\|\rho(M) f-\widetilde{\rho(M) f}\|_{\infty}+\left\|\widetilde{\rho(M) f}-(\rho(M) f)_{\Lambda}\right\|_{\infty}
$$

for all $M \in G$ and $f \in L^{2}\left(\mathbb{R}^{d}\right) \cap L^{\infty}\left(\mathbb{R}^{d}\right)$, it becomes apparent that we need to control the growth of each one of the terms in the right-hand side of the previous inequality as $M$ varies. The first of the two terms is known as reconstruction error while the other is called truncation error. Throughout the rest of the section we assume $f \in L^{2}\left(\mathbb{R}^{d}\right)$ and $\hat{f} \in L^{1}\left(\mathbb{R}^{d}\right)$.

There is an abundance of work on the study of decay estimates of these two types of errors e.g. [28, 45, 16, 6, 5, 65, 49, 30] and of the approximation error as well in one and multi-dimensions both in the context of linear (when $\Lambda=\prod_{s=1}^{d}\left[-N_{s}, N_{s}\right]$ ), non-linear and 
$n$-term approximation e.g. [35, 68, 67, 69, 71, 70]. An excellent tutorial on non-linear approximations [17] provides several more references that we did not include in this paper. The novel concept we introduce in this section is that the proper selection of approximants should take into account the variations of an image due to the action of groups of orthogonal transformations on it, e.g. rotations. This kind of variation affects the rate of convergence of linear approximations as we demonstrate with an example at the end of the section. As Table 4.2 indicates that non-linear and $n$-term approximations may be affected as well as the high-pass content of the image increases due to its rotation and the non-isotropy of the analysis kernel. In particular, if we keep $\Lambda$ fixed, the error $\left\|\rho(M) f-(\rho(M) f)_{\Lambda}\right\|$ in any relevant norm may vary with $M$. Nevertheless, the error estimate provided by Theorem 4.1 provides a search range for $\Lambda$ that does not depend on the individual transformations $M$ but it rather depends on the group to which $M$ belong to.

Before, continuing with the analysis of both errors we give an example demonstrating the practical significance of this problem in images acquired by confocal microscopy, when $\phi_{a}$ is anisotropic.

The most common practice among neuroscientists is to acquire their data by using an anisotropic sampling grid of the form $\mathbb{Z}^{2} \times(N \mathbb{Z})$, where $N=3,4$ [18], which amounts to using anisotropic analysis and synthesis kernels. The use of this grid saves time and overcomes limitations due to the quantum nature of light, but reduces the resolution to the point that spine volumes cannot be accurately estimated [18]. Scanning time increases nonlinearly [18] as the resolution in the $z$-direction increases and at $x y$ is kept high.

Heuristic methods, popular among neuroscientists have been proposed to resolve this issue [18] but those methods ignore the real mathematical problem, the undersampling in the $z$ direction. Fig. 2 shows exactly how the volumes of spines are consistently ignored in the binary segmentation of a hippocampal CA1-neuron.

Let us now return to our analysis. Take $0<\varepsilon<1, \Omega \subseteq \mathbb{T}^{d}$ and $f \in \mathcal{B}_{\Omega}^{\varepsilon}$. We also assume that $\phi_{a}$ and $\phi_{s}$ are analysis and synthesis kernels satisfying Properties (1) through (7), $\Omega \subset B_{a}^{\circ}, B_{a} \subset B_{s}^{\circ}$, and $B_{s} \subset\left(\mathbb{T}^{d}\right)^{\circ}$. By taking the Fourier transform on both sides of Eq. (2) we obtain

$$
\widehat{\tilde{f}}(\xi)=\left(\sum_{n \in \mathbb{Z}^{d}}\left\langle f, T_{n} \phi_{a}\right\rangle e^{-2 i \pi n \cdot \xi}\right) \widehat{\phi}_{s}(\xi):=A(\xi) \widehat{\phi}_{s}(\xi),
$$

where $A$ is a $\mathbb{Z}^{d}$-periodic function verifying

$$
A(\xi)=\left(\sum_{n \in \mathbb{Z}^{d}}\left\langle f, T_{n} \phi_{a}\right\rangle e^{-2 i \pi n \cdot \xi}\right)=\sum_{\ell \in \mathbb{Z}^{d}} \widehat{f}(\xi+\ell) \overline{\widehat{\phi}_{a}(\xi+\ell)} .
$$

The next observation is critical for estimating the error bounds.

4.1. Bounds for the Coefficients of $A$. Several of the estimates that will be given below critically depend on the coefficients $\left\langle f, T_{n} \phi_{a}\right\rangle$. By Parseval's Theorem, we have

$$
\left\langle f, T_{n} \phi_{a}\right\rangle=\left\langle\widehat{f}, e^{-2 \pi i n \cdot(\cdot)} \widehat{\phi}_{a}\right\rangle=\int_{\mathbb{R}^{d}} \widehat{f}(\xi) \overline{\widehat{\phi}_{a}(\xi)} e^{2 \pi i n \cdot \xi} \mathrm{d} \xi=\left(\widehat{f} \overline{\phi_{a}}\right)^{\vee}(n) .
$$




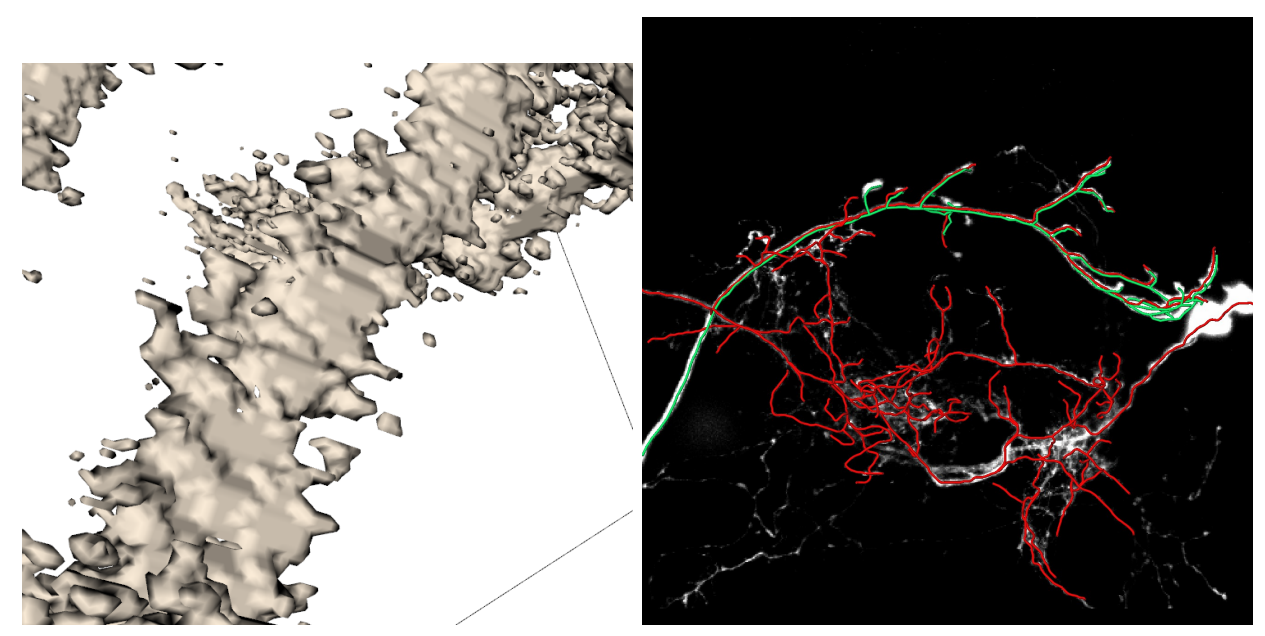

FIGURE 2. Left: Part of the binary segmentation of the Hippocampus CA1neuron shown in Fig. 1. Notice the smoothness on the dendrite's side while its 'other side' is rougher. The smoothness on the smooth side is due to the undersampling in the $z$-direction. This data set was sampled on the grid $\mathbb{Z}^{2} \times$ $(4 \mathbb{Z})$. Right: MIP results of the tracing of an olphactory cell (OP2) from the Diadem competition data sets. The centerline annotated by an expert is marked with green. Centerline tracing results with ORION are marked with red. The raw image is in the background. There are two cells in this image stack although only a single cell should have been included in the image stack. ORION traces both of them but by default it considers them as a single cell.

In general, for a function $g$ such that $\widehat{g} \in C^{2 m}\left(\mathbb{R}^{d}\right)$, where $m=1,2, \ldots$, and all of its derivatives are integrable we have

$$
\left(\Delta^{m} \widehat{g}\right)^{\vee}(x)=(2 \pi i)^{2 m}\left(x_{1}^{2}+\cdots+x_{d}^{2}\right)^{m} g(x)=(2 \pi i)^{2 m}\|x\|_{2}^{2 m} g(x) .
$$

Since, properties (1), (2) and the definition of $\mathcal{B}_{\Omega}^{\varepsilon}$ imply that the distributional Laplacian $\Delta\left(\widehat{f} \overline{\widehat{\phi}_{a}}\right)$ is integrable, we assert

$$
\left|\left\langle f, T_{n} \phi_{a}\right\rangle\right|=\left|\left(\widehat{f} \overline{\widehat{\phi}_{a}}\right)^{\vee}(n)\right| \leq \frac{\left\|\Delta\left(\widehat{f} \widehat{\phi}_{a}\right)\right\|_{1}}{(2 \pi)^{2}\|n\|_{2}^{2}}
$$

Therefore, $\sum_{n \in \mathbb{Z}^{d}}\left|\left\langle f, T_{n} \phi_{a}\right\rangle\right|<\infty$ and $A$ belongs to $A\left(\mathbb{T}^{d}\right)$.

So, if in addition to the previous assumptions for $f$ and for the analysis kernel $\phi_{a}$, we have $\hat{f} \in W^{1,2 m}$ and all partial derivatives of $\phi_{a}$ up to order $2 m$, where $m=1,2, \ldots$, are bounded, then

$$
(2 \pi)^{2 m}\|x\|_{2}^{2 m}\left|\left(\widehat{f} \widehat{\phi}_{a}\right)^{\vee}(x)\right| \leq\left\|\left(\Delta^{m}\left(\widehat{f} \overline{\widehat{\phi}_{a}}\right)\right)^{\vee}\right\|_{\infty} \leq\left\|\Delta^{m}\left(\widehat{f} \overline{\widehat{\phi}_{a}}\right)\right\|_{1},
$$


therefore, if $x \in \mathbb{R}^{d}$

$$
\left|\left\langle f, T_{x} \phi_{a}\right\rangle\right|=\left|\left(\widehat{f \widehat{\phi}_{a}}\right)^{\vee}(x)\right| \leq \frac{\left\|\Delta^{m}\left(\widehat{f} \widehat{f}_{a}\right)\right\|_{1}}{(2 \pi)^{2 m}\|x\|_{2}^{2 m}}
$$

4.2. Estimation of the Approximation Error $\left\|f-f_{\Lambda}\right\|_{\infty}$. First, we proceed with the estimation of $\|\widehat{f}-\widehat{\tilde{f}}\|_{1}$. Note that

$$
\begin{aligned}
\|\widehat{f}-\widehat{\tilde{f}}\|_{1} & =\int_{\mathbb{R}^{d}}\left|\widehat{f}(\xi)-A(\xi) \widehat{\phi}_{s}(\xi)\right| \mathrm{d} \xi \\
& =\int_{\mathbb{T}^{d}}\left|\widehat{f}(\xi)-A(\xi) \widehat{\phi}_{s}(\xi)\right| \mathrm{d} \xi+\int_{\left(\mathbb{T}^{d}\right)^{c}}\left|\widehat{f}(\xi)-A(\xi) \widehat{\phi}_{s}(\xi)\right| \mathrm{d} \xi .
\end{aligned}
$$

Using Property (6) and the fact $f \in \mathcal{B}_{\Omega}^{\varepsilon}$ the second term in the previous sum can be bounded by:

$$
\begin{aligned}
\int_{\left(\mathbb{T}^{d}\right)^{c}}\left|\widehat{f}(\xi)-A(\xi) \widehat{\phi}_{s}(\xi)\right| \mathrm{d} \xi & \leq \int_{\left(\mathbb{T}^{d}\right)^{c}}|\widehat{f}(\xi)| \mathrm{d} \xi+\sup _{\mathbb{R}^{d}}|A(\xi)| \int_{\left(\mathbb{T}^{d}\right)^{c}}\left|\widehat{\phi}_{s}(\xi)\right| \mathrm{d} \xi \\
& \leq \varepsilon\|\widehat{f}\|_{1}+\left(\sum_{n \in \mathbb{Z}^{d}}\left|\left\langle f, T_{n} \phi_{a}\right\rangle\right|\right) \varepsilon .
\end{aligned}
$$

Next we estimate the contribution of the term $\int_{\mathbb{T}^{d}}|\widehat{f}(\xi)-\widehat{\tilde{f}}(\xi)| \mathrm{d} \xi$ to the reconstruction error of $f$ :

$$
\begin{aligned}
& \int_{\mathbb{T}^{d}}\left|\widehat{f}(\xi)-A(\xi) \widehat{\phi}_{s}(\xi)\right| \mathrm{d} \xi=\int_{\mathbb{T}^{d}}\left|\widehat{f}(\xi)-\left(\sum_{\ell \in \mathbb{Z}^{d}} \widehat{f}(\xi+\ell) \overline{\widehat{\phi}_{a}(\xi+\ell)}\right) \widehat{\phi}_{s}(\xi)\right| \mathrm{d} \xi \\
& \leq \int_{\mathbb{T}^{d}}\left|\left(1-\overline{\widehat{\phi}_{a}(\xi)} \widehat{\phi}_{s}(\xi)\right) \widehat{f}(\xi)\right| \mathrm{d} \xi+\int_{\mathbb{T}^{d}}\left|\sum_{\ell \in \mathbb{Z}^{d} \backslash\{\mathbf{0}\}} \widehat{f}(\xi+\ell) \overline{\widehat{\phi}_{a}(\xi+\ell)} \widehat{\phi}_{s}(\xi)\right| \mathrm{d} \xi .
\end{aligned}
$$

Now,

$$
\begin{gathered}
\int_{\mathbb{T}^{d}}\left|\sum_{\ell \in \mathbb{Z}^{d} \backslash\{\mathbf{0}\}} \widehat{f}(\xi+\ell) \overline{\widehat{\phi}_{a}(\xi+\ell)} \widehat{\phi}_{s}(\xi)\right| \mathrm{d} \xi \leq(1+\varepsilon) \varepsilon \sum_{\ell \in \mathbb{Z}^{d} \backslash\{\mathbf{0}\}} \int_{\mathbb{T}^{d}}|\widehat{f}(\xi+\ell)| \mathrm{d} \xi \\
\leq(1+\varepsilon) \varepsilon^{2}\|\widehat{f}\|_{1} \leq 2 \varepsilon\|\widehat{f}\|_{1} .
\end{gathered}
$$

Using Property (3) we infer $\left|1-{\widehat{\widehat{\phi}_{a}(\xi)}}_{\phi_{s}}(\xi)\right| \leq 1+\left|\overline{\widehat{\phi}_{a}(\xi)} \widehat{\phi}_{s}(\xi)\right| \leq 1+(1+\varepsilon)^{2} \leq 5$. On the other hand, if $\xi \in \Omega$, Properties (4) and (5) imply $\left|1-{\widehat{\phi_{a}}}_{(\xi)} \widehat{\phi}_{s}(\xi)\right|<2 \varepsilon+\varepsilon^{2}<3 \varepsilon$. Therefore

$$
\int_{\mathbb{T}^{d}}\left|\left(1-\overline{\widehat{\phi}_{a}(\xi)} \widehat{\phi}_{s}(\xi)\right) \widehat{f}(\xi)\right| \mathrm{d} \xi=\int_{\Omega}\left|\left(1-\overline{\widehat{\phi}_{a}(\xi)} \widehat{\phi}_{s}(\xi)\right) \widehat{f}(\xi)\right| \mathrm{d} \xi+\int_{\mathbb{T}^{d} \backslash \Omega}\left|\left(1-\overline{\widehat{\phi}_{a}(\xi)} \widehat{\phi}_{s}(\xi)\right) \widehat{f}(\xi)\right| \mathrm{d} \xi
$$




$$
\leq 3 \varepsilon \int_{\Omega}|\widehat{f}(\xi)| \mathrm{d} \xi+5 \int_{\mathbb{T}^{d} \backslash \Omega}|\widehat{f}(\xi)| \mathrm{d} \xi \leq 8 \varepsilon\|\widehat{f}\|_{1}
$$

Collecting terms we conlcude,

$$
\|f-\tilde{f}\|_{\infty} \leq\left[\left(\sum_{n \in \mathbb{Z}^{d}}\left|\left\langle f, T_{n} \phi_{a}\right\rangle\right|\right)+11\|\widehat{f}\|_{1}\right] \varepsilon
$$

Now, let $\Lambda$ be a finite subset of $\mathbb{Z}^{d}$. Then,

$$
\left\|\tilde{f}-f_{\Lambda}\right\|_{\infty} \leq\left(\sum_{n \notin \Lambda}\left|\left\langle f, T_{n} \phi_{a}\right\rangle\right|\right)\left\|\hat{\phi}_{s}\right\|_{1} .
$$

Next, take $G$ to be a group of orthogonal transformations acting on $\mathbb{R}^{d}$ and $M \in G$. If $\rho(M) f \in \mathcal{B}_{\Omega}^{\varepsilon}$ then, Eqs. (4), (8) and (9) imply that for every $\Lambda$ be a finite subset of $\mathbb{Z}^{d}$ we have

$$
\begin{gathered}
\left\|\rho(M) f-(\rho(M) f)_{\Lambda}\right\|_{\infty} \\
\leq\left[\left(\sum_{n \in \mathbb{Z}^{d}}\left|\left\langle\rho(M) f, T_{n} \phi_{a}\right\rangle\right|\right)+11\|\widehat{f}\|_{1}\right] \varepsilon+\left(\sum_{n \notin \Lambda}\left|\left\langle\rho(M) f, T_{n} \phi_{a}\right\rangle\right|\right)\left\|\hat{\phi}_{s}\right\|_{1} \\
=\left[\left(\sum_{n \in \mathbb{Z}^{d}}\left|\left\langle f, T_{M n} \rho\left(M^{T}\right) \phi_{a}\right\rangle\right|\right)+11\|\widehat{f}\|_{1}\right] \varepsilon+\left(\sum_{n \notin \Lambda}\left|\left\langle f, T_{M n} \rho\left(M^{T}\right) \phi_{a}\right\rangle\right|\right)\left\|\hat{\phi}_{s}\right\|_{1} .
\end{gathered}
$$

Assuming $\hat{f} \in W^{1,2 m}$ and that $\hat{\phi}_{a}$ has bounded partial derivatives up to order $2 m$ with $m \geq 1$ Eq. (7) gives

$$
\left|\left\langle f, T_{M n} \rho\left(M^{T}\right) \phi_{a}\right\rangle\right| \leq \frac{\left\|\Delta^{m}\left(\widehat{f} \overline{\rho\left(M^{T}\right) \widehat{\phi}_{a}}\right)\right\|_{1}}{(2 \pi)^{m}\|M n\|_{2}^{m}}, \quad n \in \mathbb{Z}^{d} .
$$

Since, $\|M n\|=\|n\|$ for all grid points $n$, we conclude that the estimate of the error $\left\|\rho(M) f-(\rho(M) f)_{\Lambda}\right\|_{\infty}$ provided above depends on the norm $\left\|\Delta^{m}\left(\widehat{f} \overline{\rho\left(M^{T}\right) \widehat{\phi}_{a}}\right)\right\|_{1}$, which depends on $M$.

We can now summarize the previous discussion in the following theorem.

Theorem 4.1. Assume that $G$ is a group of orthogonal transformations acting on $\mathbb{R}^{d}, \Omega \subseteq$ $\left(\mathbb{T}^{d}\right)^{\circ}, 0<\varepsilon<1$. Suppose, $M(\Omega) \subseteq \Omega$ for all $M \in G$. We also assume that $\phi_{a}$ and $\phi_{s}$ are analysis and synthesis kernels satisfying Properties (1) through (7), $\Omega \subset B_{a}^{\circ}, B_{a} \subset B_{s}^{\circ}$, and $B_{s} \subset\left(\mathbb{T}^{d}\right)^{\circ}$ and $\rho(M) \phi_{a}=\phi_{a}$ for all $M \in G$. In addition, we assume that $\hat{\phi}_{a}$ has bounded partial derivatives up to order $2 m$ with $m \in \mathbb{Z}^{+}$. Then, for every $f \in \mathcal{B}_{\Omega}^{\varepsilon}$ such that $\hat{f} \in W^{1,2 m}$ the following estimate holds:

$$
\left\|\rho(M) f-(\rho(M) f)_{\Lambda_{N}}\right\|_{\infty} \leq C_{1} \varepsilon+C_{2} \sum_{\|n\|_{2} \geq N}\|n\|_{2}^{-2 m}, \quad M \in G,
$$

where $\Lambda_{N}=\prod_{s=1}^{d}\left[-N_{s}, N_{s}\right]$ with $N_{s}=N$ for all $s=1,2, \ldots, d$. The constants $C_{1}$ and $C_{2}$ depend only on $f$. 
Proof. First, observe that $M(\Omega) \subseteq \Omega$ for all $M \in G$ implies that, if $f \in \mathcal{B}_{\Omega}^{\varepsilon}$ then, $\rho(M) f \in$ $\mathcal{B}_{\Omega}^{\varepsilon}$. Since, $\rho(M) \phi_{a}=\phi_{a}$ for all $M \in G$ using $[10)$ we conclude

$$
\left|\left\langle f, T_{M n} \rho\left(M^{T}\right) \phi_{a}\right\rangle\right| \leq \frac{\left\|\Delta^{m}\left(\widehat{f} \overline{\phi_{a}}\right)\right\|_{1}}{(2 \pi)^{m}\|n\|_{2}^{m}}, \quad n \in \mathbb{Z}^{d} .
$$

So, the factors $C_{1}:=\left(\sum_{n \in \mathbb{Z}^{d}}\left|\left\langle f, T_{M n} \rho\left(M^{T}\right) \phi_{a}\right\rangle\right|\right)+11\|\widehat{f}\|_{1}$ and $C_{2}:=(2 \pi)^{-m}\left\|\hat{\phi}_{s}\right\|_{1}\left\|\Delta^{m}\left(\widehat{f} \overline{\hat{\phi}_{a}}\right)\right\|_{1}$ depend only on $f$. The conclusion follows from $(10)$ and

$$
\begin{aligned}
\left\|\rho(M) f-(\rho(M) f)_{\Lambda}\right\|_{\infty} & \leq\left[\left(\sum_{n \in \mathbb{Z}^{d}}\left|\left\langle f, T_{M n} \rho\left(M^{T}\right) \phi_{a}\right\rangle\right|\right)+11\|\widehat{f}\|_{1}\right] \varepsilon \\
& +\left(\sum_{n \notin \Lambda_{N}}\left|\left\langle f, T_{M n} \rho\left(M^{T}\right) \phi_{a}\right\rangle\right|\right)\left\|\hat{\phi}_{s}\right\|_{1} \cdot \leq C_{1} \varepsilon+C_{2} \sum_{\|n\|_{2} \geq N}\|n\|_{2}^{-2 m}
\end{aligned}
$$

since $M$ preserves norms.

Remark 4.2. 1) Theorem 4.1 requires analysis kernels to be invariant under the action of the groups of orthogonal transformations that may affect an image of interest. A slight modification of the statement of Theorem 4.1 can make it applicable to data representations defined by families of analysis and synthesis kernels instead of a single pair of kernels. Popular examples of these families are shearlets [34, 25] and curvelets [9]. Condition $\rho(M) \phi_{a}=\phi_{a}$ for all $M \in G$ is now replaced by the requirement that the family of analysis filters must remain invariant under the action of $G$. In other words, if one $\phi_{a}$ works well for $f$ then $\rho\left(M^{T}\right) \phi_{a}$ must be another analysis filter in the family of filters used by the data representation to allow to maintain control over the size of $\Lambda$ when approximating $\rho(M) f$ by $(\rho(M) f)_{\Lambda}$ and thus maintain the sparsity of the representation.

2) The hypothesis $M(\Omega) \subseteq \Omega$ for all $M \in G$ in the statement of the previous theorem is not redundant. Indeed, assume that $\phi_{a}$ and $\phi_{s}$ satisfy all of the assumptions of the previous theorem. In addition, we assume $B_{s}=\mathbb{T}^{2}$ and that $\hat{\phi}_{a}$ vanishes outside $\mathbb{T}^{2}$. Take $0<d<$ $\frac{\sqrt{3-2 \sqrt{2}}}{2 \sqrt{2}}$ and assume that $B_{a}=\left[-\frac{1}{2}+d, \frac{1}{2}-d\right]^{2}$ and $\Omega=B_{a}^{\circ}$. Now pick $f$ so that $\hat{f}$ is smooth and vanishes outside $\left[\frac{\sqrt{2}}{4}, \frac{1}{2}-d\right] \times\left[\frac{\sqrt{2}}{4}, \frac{1}{2}-d\right]$. If $M$ is the rotation by $\pi / 4$ then, $(\rho(M) \hat{f}) \hat{\phi}_{a}=0$, therefore $\left\langle\rho(M) f, T_{n} \phi_{a}\right\rangle=0$, for all $n \in \mathbb{Z}^{2}$. In this case $\| \rho(M) f-$ $(\rho(M) f)_{\Lambda}\left\|_{\infty}=\right\| f \|_{\infty}$ for every $\Lambda \subset \mathbb{Z}^{2}$.

We close this section with a simulation intending to demonstrate how rotations affect the rate of decay of $\left\|\rho(M) f-(\rho(M) f)_{\Lambda_{N}}\right\|_{\infty}$ as $N \rightarrow \infty$.

Let $M$ be a rotation by $\pi / 4$ in $\mathbb{R}^{2}$. Consider $f$ such that $\widehat{f}\left(\xi_{1}, \xi_{2}\right)=\chi_{I_{1}}\left(\xi_{1}\right) \chi_{I_{y}}\left(\xi_{2}\right)$, where $I_{1}=\left[-\sigma_{1},-\sigma_{2}\right] \cup\left[\sigma_{2}, \sigma_{1}\right]$ and $I_{2}=\left[-\sigma_{3}, \sigma_{3}\right]$. Let

$$
\widehat{\phi}_{a}\left(\xi_{1}, \xi_{2}\right)=e^{-\frac{\xi_{1}^{2}}{2 \sigma_{4}^{2}}-\frac{\xi_{2}^{2}}{2 \sigma_{5}^{2}}} \quad \text { and } \widehat{\phi}_{s}\left(\xi_{1}, \xi_{2}\right)=e^{-\frac{\left(\xi_{1}^{2}+\xi_{2}^{2}\right)}{2 \sigma_{6}^{2}}},,
$$

where $0 \leq \sigma_{2} \leq \sigma_{1} \leq \sigma_{4} \leq \sigma_{6} \leq 1$ and $0 \leq \sigma_{3} \leq \sigma_{5} \leq \sigma_{6}$. In this example we set $\sigma_{6}=0.6, \sigma_{5}=0.55, \sigma_{2}=0.15, \sigma_{4}=0.5, \sigma_{1}=0.25, \sigma_{3}=0.12$. We compute the errors 
$\left\|\rho(M) f-(\rho(M) f)_{\Lambda_{N}}\right\|_{\infty}$ and $\left\|f-f_{\Lambda_{N}}\right\|_{\infty}$, as $N$ grows, where $\Lambda_{N}=\left\{(m, n) \in \mathbb{Z}^{2}\right.$ : $\max (|m|,|n|) \leq N\}$.

\begin{tabular}{|c|c|c|c|}
\hline$N$ & $\frac{\left\|\rho(M) f-(\rho(M) f)_{\Lambda_{N}}\right\|_{\infty}}{\left\|f-f_{\Lambda_{N}}\right\|_{\infty}}$ & $\left\|f-f_{\Lambda_{N}}\right\|_{\infty}$ & $\left\|\rho(M) f-(\rho(M) f)_{\Lambda_{N}}\right\|_{\infty}$ \\
\hline 35 & $1.3023 \cdot 10^{15}$ & $6.3961 \cdot 10^{-31}$ & $8.3299 \cdot 10^{-16}$ \\
45 & $2.0835 \cdot 10^{163}$ & $3.8408 \cdot 10^{-180}$ & $8.0026 \cdot 10^{-17}$ \\
50 & $2.6247 \cdot 10^{267}$ & $1.2856 \cdot 10^{-298}$ & $3.3746 \cdot 10^{-31}$ \\
55 & $\infty$ & 0 & $1.3558 \cdot 10^{-108}$ \\
60 & $\infty$ & 0 & $2.8357 \cdot 10^{-228}$ \\
65 & undefined & 0 & 0 \\
\hline
\end{tabular}

In Table 4.2 we can observe the results of this experiment. Notice that $(\rho(M) f)_{\Lambda_{N}}$ converges to zero more slowly than $f_{\Lambda_{N}}$.

\section{Construction of Synthetic Tubular 3D-Data Sets}

As mentioned above, a fundamental step in the development of a computational platform for neuronal reconstructions is the segmentation of the dendritic arbor and the extraction of its centerline. Validating the accuracy of the performance of these two tasks heavily relies on the manual segmentation of dendritic arbors which is time consuming, tedious and often quite subjective. Therefore, the benchmarking of dendritic arbor segmentation and centerline extraction algorithms often becomes controversial, as most of the times the 'gold standard' entirely relies on the experience of the user and cannot be verified against histology. Indeed, it is very common in neuroscience imaging that segmentation results manually obtained by experts working on the same data sets significantly differ, and automatic segmentation algorithms may outperform experts. Hence, there is a real need for highly accurate computational phantoms representing tubular structures in 3D that can be used to benchmark the baseline performance of segmentation and morphological reconstruction algorithms. To this end, we introduce a new method for the construction of highly accurate computational phantoms that represent the geometry of realistic tubular 3D-structures. Our method yields very complex 3D-data sets emulating with high accuracy at the resolution level normally used in confocal microscopy the prescribed morphological properties: centerline, branching points and branch diameter. Thus such data sets enable the reliable validation of segmentation and centerline extraction algorithms. It is clear that noisy data sets can easily be derived from our algorithm using standard methods like those in [73].

One feature of our approach is the ability to simulate varying fluorescence intensity values even within the same cross-section of the volume. The basic scenario for the spatial distribution of the fluorescence intensity values assumes that at any cross-section the maximum intensity occurs only at centerline voxel. The intensity values for each voxel in a crosssection perpendicular to the centerline (transversal cross-section) decreases almost radially, in the sense that voxels in the same transversal cross-section and equidistant from the centerline voxel have the same intensity values. Moreover, for any two transversal cross-sections whose centerline voxels have the same intensity values, the spatial distributions of the intensity values in these cross-sections are identical. We refer to this model of spatial distribution 
of intensity values as the ideal tubular intensity distribution model. This radial symmetry of the intensity function can only be implemented approximately in a digital phantom, since voxels are not dimensionless in the 3D space. Moreover, the centerline must be smooth everywhere except possibly at branching points.

5.1. Related Work. Only few methods to generate synthetic data for tubular objects can be found in the literature. In particular, Canero et al. [10] proposed a method to generate images of synthetic vessels. After generating a random centerline, the intensity for each pixel in the vessel is modeled as a function of the distance from the pixel to the centerline and the radius of the vessel. Vasilkoski et al. [73] proposed a method to generate a 3D image stack of a neuron assuming that the infused fluorophore is distributed uniformly throughout the neuron and that the background intensity is zero. Then, they convolve the volume with a Gaussian point spread function to simulate the photon count $\mu(x, y, z)$ in the image stack. The actual photon count $n(x, y, z)$ for each voxel was generated randomly generated using the Poisson distribution with mean equal to $\mu(x, y, z)$. Bouix et al. [7] created synthetic tubular structures by using a predefined centerline. They slide a sphere centered on the centerline starting at a seed point. Voxel intensities are all the same inside the tubular volume while noise may be added at the final stage. Unfortunately, all these methods tend to suffer from a significant degree of geometric artifacts.

5.2. Methods. The first step in our approach, to create synthetic data volumes such as dendritic arbor phantoms is to construct the prescribed volume at a very high spatial resolution level, much higher than the resolution level used in confocal microscopy. Next, to reduce the resolution of those volumes, we downsample the data by a factor of two per dimension. To bring the data set to the desired resolution level we typically repeat the downsampling step as needed (typically three or four times). The problem that often arises with this reduction of resolution is aliasing causing image degradation with errors directionally varying in $3 D$. This problem compromises the radial symmetry of the ideal tubular intensity distribution model. To reduce the effect of such aliasing we first filter the input volume with a low-pass antialiasing filter. One might naturally wonder what are the properties that the antialiasing filters should have in order to minimize the adverse effects of this reduction of resolution on the symmetry properties imposed by the ideal tubular intensity distribution model. Although we do not directly address this question, we will provide below a family of antialiasing filters and justify why they are suitable for this application by invoking Theorem 4.1. We are now ready to proceed with the description of our approach.

Using the prescribed geometric properties (i.e., centerline points, branching and terminal points and thickness) of the sought tubular structure (e.g., a dendrite) we first create a very high resolution approximation of the desired volume in the physical domain. In the language of multiscale analysis, this high resolution image provides an approximation of the physical structure at a very high scale and, so, it may not be distinguished from the prototype structure living in the physical 'continuous' domain $\mathbb{R}^{3}$. We denote this initial volume by $I_{0}$. To create the centerline of $I_{0}$ we use cubic spline interpolation in 3D. Using this centerline and the diameter information we create a 'mask' $M_{0}$ which is an indicator function taking two values only, 0 if a voxel does not belong to $I_{0}$ and 1 otherwise. To create $M_{0}$ we superimpose spheres of radii matching the diameter of $I_{0}$ at the location where the sphere is centered. The 
centers of these spheres belong to the centerline of $I_{0}$. The centers of these spheres are not uniformly distributed on the centerline of $I_{0}$. Their distribution varies depending on the spatial accuracy needed for $M_{0}$ (Figure 3(a)). The use of spheres helps to successfully and accurately create the curved parts of $M_{0}$ with low computational cost. Each voxel in the interior of each of one of these spheres is assigned the value 1 (Figure 3(b)). Thus, $M_{0}$ is defined to be characteristic function of the set of voxels whose value is at least equal to 1 . To algorithmically define the transversal cross-sections in $I_{0}$, for each voxel $\mathbf{v}$ with mask value $M_{0}(\mathbf{v})=1$ we assign a 'tag', $\mathbf{c}(\mathbf{v})$, where $\mathbf{c}(\mathbf{v})$ is the most proximal centerline voxel to $\mathbf{v}$. We thus partition $I_{0}$ into cross-sections via the equivalence relation $\mathbf{v}_{\mathbf{1}} \sim \mathbf{v}_{\mathbf{2}}$ if and only if $\mathbf{c}\left(\mathbf{v}_{\mathbf{1}}\right)=\mathbf{c}\left(\mathbf{v}_{\mathbf{2}}\right)$. Figure $3(\mathrm{~d})$ depicts how this equivalence relation defines cross-sections in a digital high resolution volume $I_{0}$. So far, the intensity values of $I_{0}$ are identical with the values of $M_{0}$.

To complete the construction of $I_{0}$ we assign the desired intensity values for each voxel at which $M_{0}$ is equal to 1 . For all other voxels the intensity is set constant to a fixed 'background' value. For dendritic arbor phantoms the luminosity intensity on the centerline may decay as the distance of a point in the dendrite from the soma increases in order to simulate the decaying concentration of the infused fluorophore in distal branches from the point of infusion. However, several other models may be chosen to simulate the fluorescence intensity values induced by various fluorophore administration protocols.

In our approach, we assume that both rates of decay of the intensity values, radially, in any transversal cross-section and along the centerline, are constant. However, the theoretical model of the luminosity intensity at cross-sections assumes that this function is an isotropic Gaussian. This assumption is standard across all proposed models for confocal microscopy data. Implementing, though an isotropic Gaussian in small transversal cross-sections gives results no different from those obeying the linear decay model both radially in transversal cross-section and along the centerline. Fig. 3(e) depicts an example of the luminosity intensity in a transversal cross section with radius $R=50$ pixels, background intensity $I_{B G}=10$ and maximum intensity at the center of the cross-section $I_{\max }=150$.

Figs. 4 (f-i) depicts the intensity obtained for a synthetic tubular structure: shown are images for the plane $x-y$ at $z=160,168,176,180$. The maximum intensity is obtained at $z=160$ because the centerline is on this plane. The intensity value at a voxel is high if the voxel is close to the skeleton and the intensity decays as we approach the boundary.

The original synthetic volume $I_{0}$ created so far has 8 or even 16 times higher resolution than that of a typical data set acquired using confocal microscopy. In order to generate a 3D data volume useful for our purposes we need to drastically reduce the resolution by a factor of 8 at least. Simple downsampling is the first obvious, yet bad choice. Downsampling following the application of a special antialiasing filter is the right approach. In the following, we argue about the properties of this filter that mitigate undesirable directional aliasing.

A plain cylinder in $\mathbb{R}^{3}$ can be modeled using the tensor product of two Gaussians,

$$
f_{\sigma_{1}, \sigma_{2}}(x, y, z)=e^{-\frac{x^{2}}{2 \sigma_{1}^{2}}} e^{-\frac{y^{2}+z^{2}}{2 \sigma_{2}^{2}}} x, y, z, \in \mathbb{R} .
$$

The centerline of this cylinder is the $x$-axis. We take $\sigma_{1}, \sigma_{2}>0$. The first Gaussian factor controls the length of the cylinder while the second controls the decay of the intensity values of this structure. The cylinder can be oriented to any different centerline by applying a 3D 


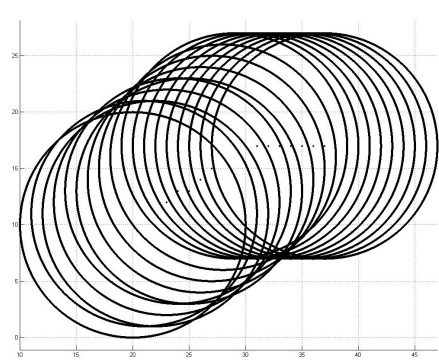

(a)

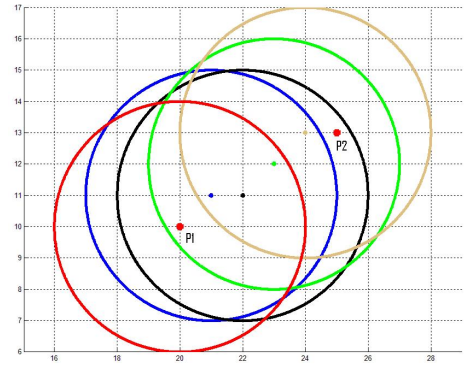

(b)

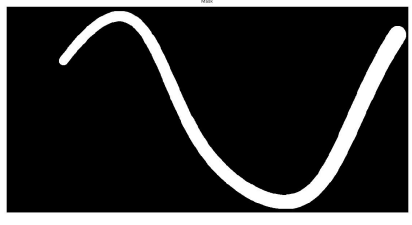

(c)

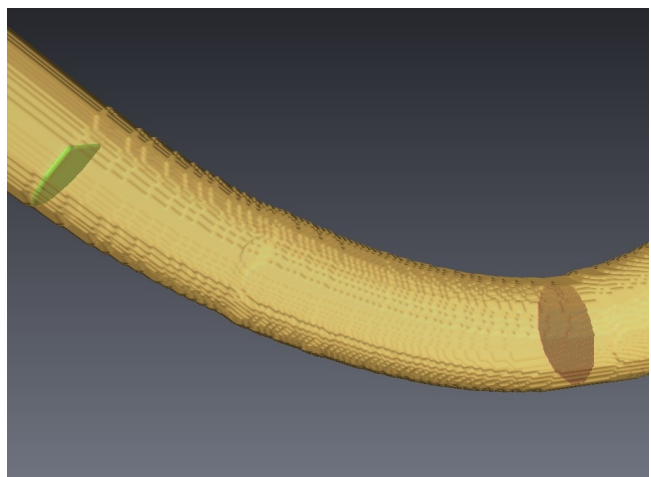

(d)

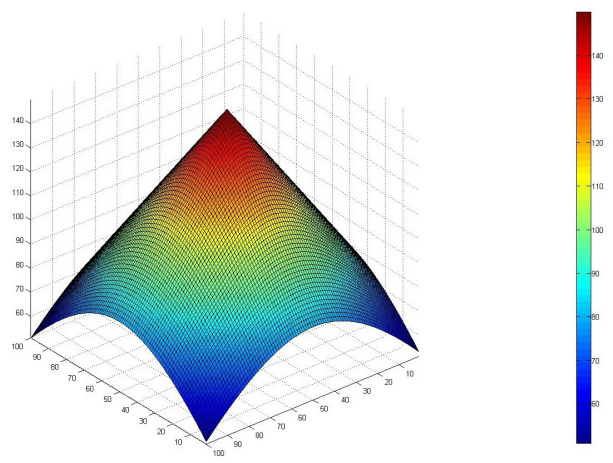

(e)

FIGURE 3. (a) Sideview of cross sections of spheres whose centers belong to the centerline. (b) A snapshot of the resulting mask $M_{0}$ from the same observation point. The small marching step of centers of the spheres yields a smooth digitized mask. (c) Binary mask with radius increasing along the centerline. (d) Circular cross section for two points on the centerline. (e) Graph of fluorescent intensity on a circular cross section.

rotation $R$ on the argument of $f$ and must be digitized in a way that, ideally, does not generate artifacts due to its spatial orientation. The original tubular structure $\mathcal{I}$ can then be considered as finite sum of the form

$$
\mathcal{I}=\sum_{i=1}^{n} \sum_{k=1}^{K} \sum_{j_{1}=1}^{J_{1}} \sum_{j_{2}=1}^{J_{2}} T_{x_{i}} R_{k} a_{i, k, j_{1}, j_{2}} f_{\sigma_{j_{1}}, \sigma_{j_{2}}}, \quad R_{k} \in S O(3), \quad a_{i, k, J_{1}, j_{2}}>0 .
$$

It is this volume defined on $\mathbb{R}^{3}$ and from this volume we essentially create $I_{0}$ by applying Theorem 4.1. Since, the rotations $R_{k}$ may be random we must assume that the set $\Omega$ the theorem requires must be invariant under all 3D-rotations. Pick a desirable $0<\varepsilon<1$. Since,

$$
\hat{f}\left(\xi_{1}, \xi_{2}, \xi_{3}\right)=(2 \pi)^{\frac{3}{2}} \sigma_{j_{1}} \sigma_{j_{2}}^{2} e^{-2 \pi^{2} \sigma_{j_{1}}^{2} \xi_{1}^{2}} e^{-2 \pi^{2} \sigma_{j_{2}}^{2}\left(\xi_{2}^{2}+\xi_{3}^{2}\right)},
$$

it is not hard to observe that $\Omega$ must contain all sets of the form $\left[-\frac{a_{j_{1}, j_{2}}}{\sigma_{j_{1}}}, \frac{a_{j_{1}, j_{2}}}{\sigma_{j_{1}}}\right] \times\left[-\frac{a_{j_{1}, j_{2}}}{\sigma_{j_{2}}}, \frac{a_{j_{1}, j_{2}}}{\sigma_{j_{2}}}\right] \times$ $\left[-\frac{a_{j_{1}, j_{2}}}{\sigma_{j_{2}}}, \frac{a_{j_{1}, j_{2}}}{\sigma_{j_{2}}}\right]$, where $a_{j_{1}, j_{2}}>0$ depends on $\varepsilon$ and all rotations of these parallilepipeds. This implies that $\Omega$ must be a sphere centered at the origin whose radius is greater than all $\frac{a_{j_{1}, j_{2}}}{\sigma_{j_{1}}}$ 


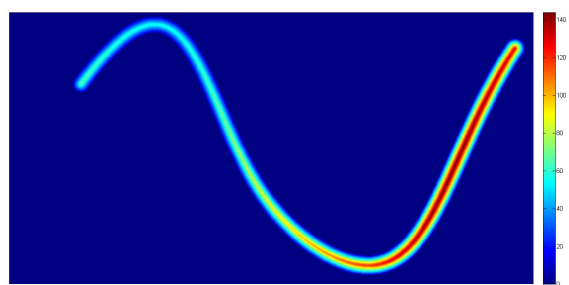

(a)

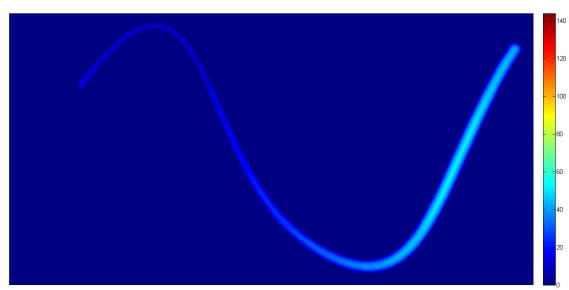

(c)

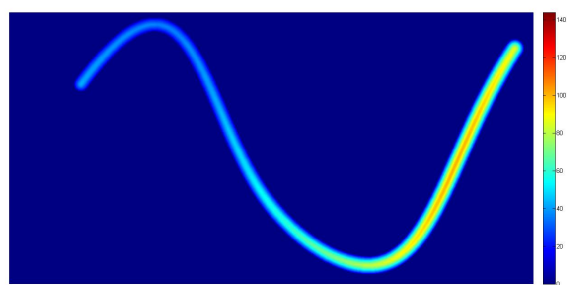

(b)

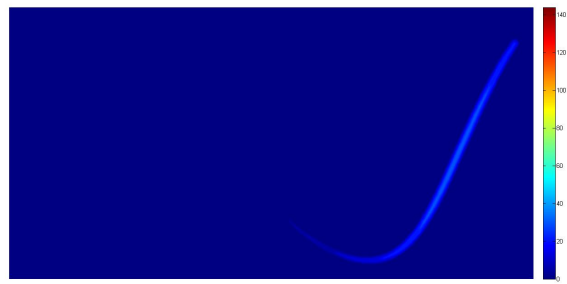

(d)

FIGURE 4. (a)-(d) Intensity values on the high resolution synthetic volume at different $x-y$ planes $(\mathrm{z}=160,168,176,180)$.

and all $\frac{a_{j_{1}, j_{2}}}{\sigma_{j_{2}}}$. Then, according to Theorem 4.1 the analysis kernel we use (theoretically only) to derive $I_{0}$ from $\mathcal{I}$ must be radial. To this end we use a refinable function $\phi_{a}$ which defines an Isotropic Multiresolution Analysis [55] which is an MRA with the additional property that each resolution space $V_{j}$ is invariant under rotations as well. The use of the MRA will soon become clear. Take

$$
\widehat{\phi^{a}}(\xi):=\left\{\begin{array}{cl}
1, & |\xi|<1 / 4 \\
\frac{1+\cos \left(6 \pi|\xi|-\frac{3 \pi}{2}\right)}{2}, & 1 / 4<|\xi|<5 / 12 \\
0, & |\xi|>5 / 12,
\end{array}\right.
$$

and consider $\phi_{a}^{j}:=2^{3 j / 2} \phi_{a}\left(2^{j} \cdot\right)$ as the analysis kernel of Theorem 4.1 where $j$ is the appropriate scale required by the theorem. Note that in this case $\Omega=B_{a}=B\left(0,2^{j} / 4\right)$. This condition determines the scale $j$. The synthesis kernel is of similar form, but we do not need it here, because the volume $I_{0}$ consists of the values $\left\{\left\langle\mathcal{I}, T_{2^{-j} n} \phi_{a}^{j}\right\rangle: n \in \mathbb{Z}^{3}\right\}$. Thus, we will make no further reference to it. There is one added benefit which we obtain for free. Since $\phi_{a}^{j}$ has compact support in the frequency domain, $I_{0}$ is covariant to translations. Simply, one does not need worry about the effect of translations in this digitization process.

The Isotropic Multiresolution Analysis allows to reduce the resolution as needed. This is where we make use of the fact that this construct is an MRA. To do so, we use as the antialiasing filter the mask $H_{0}^{a}$ of the refinable function $\phi_{a}$. This is given, in the frequency 
domain, by

$$
H_{0}^{a}(\xi)=\left\{\begin{array}{cl}
1, & |\xi|<1 / 8 \\
\frac{1+\cos \left(12 \pi|\xi|-\frac{3 \pi}{2}\right)}{2}, & 1 / 8<|\xi|<5 / 24 \\
0, & |\xi|>5 /<24 .
\end{array}\right.
$$

To summarize the previous discussion we list the steps of the proposed algorithm for generating synthetic 3D-data sets of tubular structures.

\section{Algorithm 1}

Input: Manual reconstruction of a neuron.

Output: A computational phantom of a neuron.

Step 1: Create a high resolution volume

1.1 Refine manual reconstruction: compute for each branch new centerline points using cubic interpolation.

1.2 Create neuron's shape: center a sphere at each centerline and assign value equal to 1 to each voxel inside the sphere.

1.3 Compute the intensity for each voxel: The intensity is a function of the distance from each voxel to the centerline and radius of tubular structure and it satisfies the ideal tubular intensity distribution model.

Step 2: Downsample volume

2.1 Decrease the resolution: Apply an isotropic low-pass filter, e.g. $H_{0}^{a}$ and downsample.

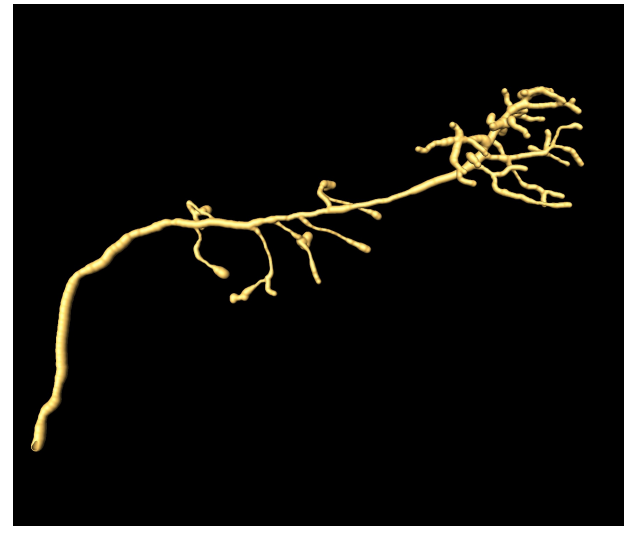

(a)

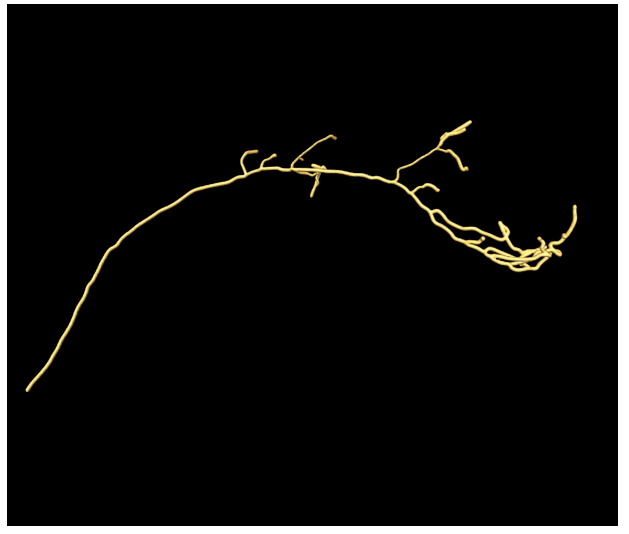

(b)

FIGURE 5. Phantoms of olfactory dendrites (OP1 and OP2) generated from information from the Diadem competition site.

5.3. Experiments. We performed two sets of experiments to illustrate our algorithm. For the first set of experiments we construct simple volumes such as straight cylinder whose centerline lies on a circle. For the second set of experiments, we constructed three synthetic dendrite volumes using specifications from the DIADEM competition. The reader can 


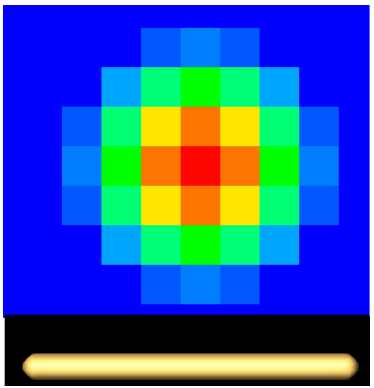

(a)

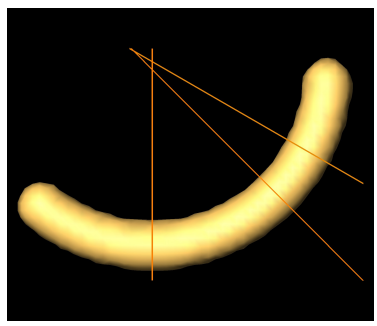

(c)

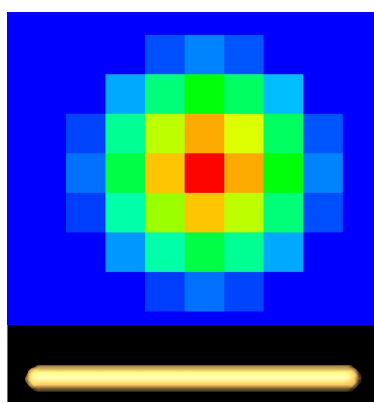

(b)

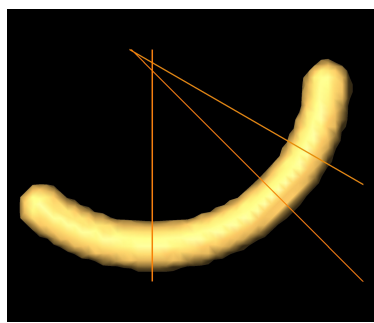

(d)

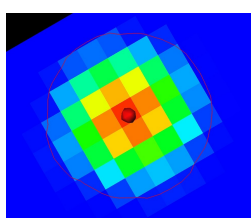

(e)

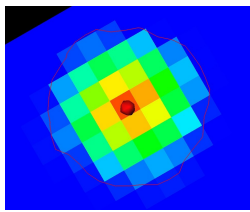

(h)

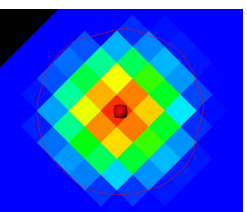

(f)

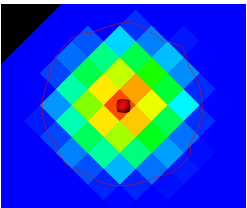

(i)

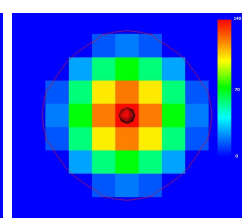

$(\mathrm{g})$

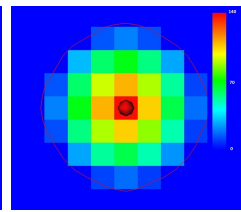

(j)

FIGURE 6. (a)(b) Depiction of a cross-section and isosurface of cylinder using our method and symlets. (c) (d) Isosurface of the second volume for first set of experiments using our method and symlets; slices at three angles $\left(30^{\circ}, 45^{\circ}\right.$, and $\left.90^{\circ}\right)$ with respect to the $\operatorname{arc}$ of the circle depicting the centerline. (e) (g) Depiction of a cross section at the slices shown on (c) by our method; (h) (j) cross section at the slices shown on (d) by symlets.

observe how the radial symmetry of the cross-sectional intensity function is achieved regardless of the incidence angle of the cross-section, due to the use of isotropic filters with small transition band, such as the proposed IMRA-filter $H_{0}^{a}$.

On these sets we evaluated the capabilities of the proposed method. We focused on the following three desirable properties. (i) The symmetry of the luminosity intensity function in every cross-section: this function must satisfy $I(n)=I(m)$ if $\|n-c\|=\|n-m\|$, where $c$ 
is the center of the cross-section. (ii) The smoothness of the centerline: in a realistic volume, the centerline must be a polygonal line. (iii) The variation of the angle of the normal vector at any point on the boundary isosurface and the centerline. Typically, this angle must be equal to $90^{\circ}$ except at bifurcation points. We used these criteria to qualitatively evaluate the performance of the proposed method using both the isotropic low-pass-IMRA filters $H$ and filters obtained from a tensor-product of 1D-symlets. It can be observed from Figure 6 that the isotropic filter performs better than the symlet filter counterpart.

Acknowledgments: This work was supported in part by NSF grants DMS 0915242, DMS 1005799 and DMS 1008900, and by NHARP grant 003652-0136-2009.

\section{REFERENCES}

[1] K.A. Al-Kofahi, S. Lasek, D.H. Szarowski, C.J. Pace, and G. Nagy. Rapid automated three-dimensional tracing of neurons from confocal image stacks. IEEE Trans. Information Technology in Biomedicine, 6(2):171-187, June 2002.

[2] G. A. Ascoli. Progress and perspectives in computational neuroanatomy. Anatomical Record, 257(6):195207, 1999.

[3] W. Bai, X. Zhou, L. Ji, J. Cheng, and S.T.C. Wong. Automatic dendritic spine analysis in two-photon laser scanning microscopy images. Cytometry A, (71A):818-826, July 2007.

[4] Wenjia Bai, Xiaobo Zhou, Liang Ji, Jie Cheng, and Stephen T. C. Wong. Automatic dendritic spine analysis in two-photon laser scanning microscopy images. Cytometry Part A, 71A(10):818-826, 2007.

[5] B.G. Bodmann, A. Melas, M. Papadakis, and T. Stavropoulos. Analog to digital revisited: Controlling the accuracy of reconstruction. Sampling Theory in Signal and Image Processing, 5(3):321-340, 2006.

[6] C. De Boor, R.A. DeVore, and A. Ron. Approximation from shift-invariant subspaces of $l_{2}\left(\mathbb{R}^{d}\right.$. Trans. Amer. Math. Soc., 341(2):787-806, 1994.

[7] S. Bouix, K. Siddiqi, and A. Tannenbaum. Flux driven automatic centerline extraction. Medical Image Analysis, 9(3):209-221, 2005.

[8] K.M. Brown, G. Barrionuevo, A.J. Canty, V.D. Paola, J.A. Hirsch, G.S.X.E. Jefferis, J. Lu, M. Snippe, I. Sugihara, and G.A. Ascoli. The DIADEM data sets: representative light microscopy images of neuronal morphology to advance automation of digital reconstructions. Neuroinformatics, 9(2-3):143-157, 2011.

[9] Emmanuel J. Candès and David L. Donoho. New tight frames of curvelets and optimal representations of objects with piecewise $C^{2}$ singularities. Comm. Pure Appl. Math., 57(2):219-266, 2004.

[10] C. Caņero and P. Radeva. Vesselness enhancement diffusion. Pattern Recognition Letters, 24(16):31413151, 2003.

[11] CBL. ORION: Online Reconstruction and functional Imaging Of Neurons, Jun. 2008.

[12] J. Cheng, X. Zhou, E. Miller, R.M. Witt, J. Zhu, B.L. Sabatini, and S.T.C. Wong. A novel computational approach for automatic dendrite spines detection in two-photon laser scan microscopy. Journal of Neuroscience Methods, 165(1):122-134, 2007.

[13] Jie Cheng, Xiaobo Zhou, Eric Miller, Veronica Alvarez, Bernardo Sabatini, and Stephen Wong. Oriented markov random field based dendritic spine segmentation for fluorescence microscopy images. Neuroinformatics, 8:157-170, 2010. 10.1007/s12021-010-9073-y.

[14] S. Choy, Kun C., Yong Z., M. Baron, M.A. Teylan, Yong K., Chong-Sze T., Zhihuan S., and S.T.C Wong. Multi scale and slice-based approach for automatic spine detection. In Engineering in Medicine and Biology Society (EMBC), 2010, pages 4765 - 4768, 2010.

[15] H. J. Curtis and K. S. Cole. Transverse electric impedance of the squid giant axon. J. General Physiol, 21:757-765, 1938.

[16] R. A. DeVore, B. Jawerth, and V. Popov. Compression of wavelet decomposition. Am. J. of Math., 114(4):737-785, 1992.

[17] R.A. DeVore. Non-linear approximation. Acta Numerica, 7:51-150, 1998. 
[18] Dani Dumitriu, Alfredo Rodriguez, and John H. Morrison. High-throughput, detailed, cell-specific neuroanatomy of dendritic spines using microinjection and confocal microscopy. Nat. Protocols, 6(9):13911411, Aug 2011.

[19] J. Fan, Zhou X., J.G. Dy, Y. Zhang, and S.T. Wong. An automated pipeline for dendrite spine detection and tracking of $3 \mathrm{~d}$ optical microscopy neuron images of in vivo mouse models. Neuroinformatics, May 2009. In Press.

[20] Jing Fan, Xiaobo Zhou, Jennifer Dy, Yong Zhang, and Stephen Wong. An automated pipeline for dendrite spine detection and tracking of $3 \mathrm{~d}$ optical microscopy neuron images of in vivo mouse models. Neuroinformatics, 7:113-130, 2009. 10.1007/s12021-009-9047-0.

[21] J.R. Glaser and E.M. Glaser. Neuron imaging with neurolucida-a pc-based system for image combining microscopy. Computerized Medical Imaging and Graphics, 14(5):307-317, 1990.

[22] G. Gonzalez, F. Fleuret, and P. Fua. Automated delineation of dendritic networks in noisy image stacks. In Proc. European Conference on Computer Vision, pages 214-227, Marseille, France, Oct. 12-18 2008.

[23] Arvind Govindarajan, Inbal Israely, Shu-Ying Huang, and Susumu Tonegawa. The dendritic branch is the preferred integrative unit for protein synthesis-dependent ltp. Neuron, 69(1):132-146, 2011.

[24] Arvind Govindarajan, Raymond J. Kelleher, and Susumu Tonegawa. A clustered plasticity model of longterm memory engrams. Nat Rev Neurosci, 7(7):575-583, Jul 2006.

[25] Kanghui Guo and Demetrio Labate. Optimally sparse multidimensional representation using shearlets. SIAM J. Math. Anal., 39, 2007.

[26] M.L. Hines and N.T. Carnevale. NEURON: a tool for neuroscientists. The Neuroscientist, 7:123-135, 2001.

[27] A. L. Hodgkin, A. F. Huxley, and B. Katz. Measurement of current-voltage relations in the membrane of the giant axon of loligo. J. Physiol., 116:424448, 1952.

[28] L. Jingfan and F. Gensun. On truncation error bound for multidimensional sampling expansion Laplace transform. Analysis in Theory and Applications, 1:52-57, 2004.

[29] B. Jowet. Plato: Theaetetus, http://ebooks.adelaide.edu.au/p/plato/p71th/index.html.

[30] Jetter K. and Plonka G. A survey on $L^{2}$-approximation order from shift-invariant spaces. In N. Dyn, D. Leviatan, D. Levin, and A. Pinkus, editors, Multivariate Approximation and Applications, pages 73111. Cambridge University Press, 2001.

[31] I.A. Kakadiaris, A. Santamaría-Pang, C. Colbert, and P. Saggau. Morphological reconstruction of living neurons. In J. Rittscher, R. Machiraju, and S.T.C. Wong, editors, Microscopic Image Analysis for Life Science Applications. Artech House Publishers, Norwood, MA, 2007.

[32] I.A. Kakadiaris, A. Santamaría-Pang, C. Colbert, and P. Saggau. Automatic 3-D morphological reconstruction of neuron cells from multiphoton images. In J. Rittscher, R. Machiraju, and S.T.C. Wong, editors, Microscopic Image Analysis for Life Science Applications, pages 389-399. Artech House, Norwood, MA, 2008.

[33] B. Katz. Nerve, Muscle and Synapse. McGraw-Hill, N.Y., 1966.

[34] D. Labate, W. Lim, G. Kutyniok, and G. Weiss. Sparse multidimensional representation using shearlets. In M. Unser, editor, Proc. Wavelets XI, volume 5914 of SPIE Proceedings, pages 247-255, January 2005.

[35] D. Leviatan and V. N. Temlyakov. Simultaneous approximation by greedy algorithms. Advances in Computational Mathematics, 25(1):73-90, June 2006.

[36] Qing Li, Xiaobo Zhou, Zhigang Deng, M. Baron, M.A. Teylan, Yong Kim, and S.T.C. Wong. A novel surface-based geometric approach for $3 \mathrm{~d}$ dendritic spine detection from multi-photon excitation microscopy images. In Proc. Biomedical Imaging: From Nano to Macro, 2009. ISBI '09. IEEE International Symposium on, pages 1255-1258, 28 2009-july 12009.

[37] B. E. Losavio and P. Saggau. Dendritic branching increases coincidence detection in neurons. Society for Neuroscience Abstracts, 32, 2006. IN FILE.

[38] B.E. Losavio, Y. Liang, A. Santamaria-Pang, I.A. Kakadiaris, C.M. Colbert, and P. Saggau. Live neuron morphology automatically reconstructed from multiphoton and confocal imaging data. Journal of Neurophysiology, 100:2422-2429, 2008. 
[39] B.E. Losavio, G.D. Reddy, C.M. Colbert, I.A. Kakadiaris, and P. Saggau. Combining optical imaging and computational modeling to analyze structure and function of living neurons. In Proc. $28^{t} h$ Annual International Conference of the IEEE Engineering in Medicine and Biology Society, volume 1, pages 668-670, New York, NY, Aug. 30 - Sep. 32006.

[40] J. Lu. Neuronal tracing for connectomic studies. Neuroinformatics, 9(2-3):159-166, 2011.

[41] J. Luisi, A. Narayanaswamy, Z. Galbreath, and B. Roysam. The farsight trace editor: an open source tool for 3-D inspection and efficient pattern analysis aided editing of automated neuronal reconstructions. Neuroinformatics, 9(2-3):305 - 315, September 2011.

[42] E. Meijering. Neuron tracing in Perspective. Cytometry Part A, 77(7):693-704, 2010.

[43] Erik Meijering. Neuron tracing in perspective. Cytometry Part A, 77A(7):693-704, 2010.

[44] A. Narayanaswamy, Y. Wang, and B. Roysam. 3-D image pre-processing algorithms for improved automated tracing of neuronal arbors. Neuroinformatics, 9(2-3):219-231, 2011.

[45] A.Y. Olenko and T.K. Pogány. A precise upper bound for the error of interpolation of stochastic processes. Theory of Probability and Math. Statistics, 71:151-163, 2005.

[46] J. van Pelt and A. Schierwagen. Morphological analysis and modeling of neuronal dendrites. Mathematical Biosciences, 188(1-2):147-155, 2004.

[47] H. Peng, Z. Ruan, D. Atasoy, and S. Sternson. Automatic reconstruction of 3D neuron structures using a graph-augmented deformable model. Bioinformatics, 26(12), 2010.

[48] Daniel Peruzzi, Muhammad Amri Abdul Karim, Susan J. Hendricks, Julie Simpson, and Jack Glaser. Spine detection and reconstruction across multiple fields-of-view with automated neuron tracing. 2007 Society for Neuroscience Meeting, 2007.

[49] G. Plonka. Approximation order provided by refinable function vectors. Constr. Approx., 13(2):221-244, 1997.

[50] Li Q. and Deng Z. A surface-based 3d dendritic spine detection approach from confocal microscopy images. IEEE, Trans. Image Processing, 2011. to appear.

[51] G. D. Reddy and P. Saggau. Development of a random-access multi-photon microscope for fast threedimensional functional recording of neuronal activity. volume 8 of Progress in Biomedical Optics and Imaging, 2007. NOT IN FILE.

[52] A. Rodriguez, D. Ehlenberger, K. Kelliher, M. Einstein, S.C. Henderson, J.H. Morrison, P.R. Hof, and S.L. Wearne. Automated reconstruction of three-dimensional neuronal morphology from laser scanning microscopy images. Methods, 30(1):94-105, 2003.

[53] A. Rodriguez, D.B. Ehlenberger, P.R. Hof, and S.L. Wearne. Three-dimensional neuron tracing by voxel scooping. Journal of Neuroscience Methods, 184(1):169-175, Oct. 2009.

[54] Hof P.R. Wearne S.L. Rodriguez A., Ehlenberger D. Rayburst sampling, an algorithm for automated three-dimensional shape analysis from laser-scanning microscopy images. Nature Protocols, 1:21562161, 2006.

[55] J.R. Romero, S.K. Alexander, S. Baid, S. Jain, and M. Papadakis. The geometry and the analytic properties of Isotropic Multiresolution Analysis. Advances in Computational Mathematics, 31:283-328, Oct. 2009.

[56] D.A. Rusakov and M.G. Stewart. Quantification of dendritic spine populations using image analysis and a tilting disector. Journal of Neuroscience Methods, 60:11-21, 1995.

[57] A. Santamaria-Pang, T.S. Bildea, C. Colbert, P. Saggau, and I.A. Kakadiaris. Towards segmentation of irregular tubular structures in 3D confocal microscope images. In Proc. MICCAI Workshop in Microscopic Image Analysis and Applications in Biology, pages 78-85, Denmark, Copenhagen, Oct. 1-6 2006.

[58] A. Santamaria-Pang, C.M. Colbert, B. Losavio, P. Saggau, and I.A. Kakadiaris. Automatic morphological reconstruction of neurons from optical images. In Proc. International Workshop in Microscopic Image Analysis and Applications in Biology, Piscataway, NJ, Sep. 3-4 2007.

[59] A. Santamaria-Pang, C.M. Colbert, P. Saggau, and I.A. Kakadiaris. Automatic centerline extraction of irregular tubular structures using probability volumes from multiphoton imaging. In Proc. Medical Image Computing and Computer-Assisted Intervention, pages 486-494, Brisbane, Australia, Oct. 29 - Nov. 2 2007. 
[60] A. Santamaria-Pang, P-H. Herrera, M. Papadakis, Saggau P., and I.A. Kakadiaris. Automatic morphological reconstruction of neurons from multiphoton and confocal microscopy images. submitted, 2011.

[61] Ascoli G.A. Scorcioni R., Polavaram S. L-measure: a web-accessible tool for the analysis, comparison and search of digital reconstructions of neuronal morphologies. Nature Protocols, 3(5):866-876, 2008.

[62] S.L. Senft. A brief history of neuronal reconstruction. Neuroinformatics, 9(2-3):119-128, 2011.

[63] Hao-wei Shen, Shigenobu Toda, Khaled Moussawi, Ashley Bouknight, Daniel S. Zahm, and Peter W. Kalivas. Altered dendritic spine plasticity in cocaine-withdrawn rats. The Journal of Neuroscience, 29(9):2876-2884, 2009.

[64] Haowei Shen, Susan Sesack, Shigenobu Toda, and Peter Kalivas. Automated quantification of dendritic spine density and spine head diameter in medium spiny neurons of the nucleus accumbens. Brain Structure and Function, 213:149-157, 2008. 10.1007/s00429-008-0184-2.

[65] T. Strohmer and J. Tanner. Implementations of Shannon's sampling theorem, a time-frequency approach. Sampl. Theory Signal Image Process., 4(1):1-17, Jan. 2005.

[66] Sharon Swanger, Xiaodi Yao, Christina Gross, and Gary Bassell. Automated 4D analysis of dendritic spine morphology: applications to stimulus-induced spine remodeling and pharmacological rescue in a disease model. Molecular Brain, 4:1-14, 2011. 10.1186/1756-6606-4-38.

[67] V. N. Temlyakov. Greedy algorithms with regard to multivariate systems with special structure. Constr. Approx., 16(3):399-425, 2000.

[68] V. N. Temlyakov. Weak greedy algorithms. Adv. Comput. Math., 12(2-3):213-227, 2000.

[69] V. N. Temlyakov. Greedy algorithms in banach spaces. Adv. Comput. Math., 14(3):277-292, 2001.

[70] J. Tropp. Algorithms for simultaneous sparse approximation. part ii: convex relaxation. Signal Processing, special issue "Sparse approximations in signal and image processing", 86:589-602, April 2006.

[71] J. Tropp, A. Gilbert, and M. Strauss. Algorithms for simultaneous sparse approximation. part I: greedy pursuit. Signal Processing, special issue "Sparse approximations in signal and image processing", 86:572-588, April 2006.

[72] C. Uehara, C. M. Colbert, P. Saggau, and I.A. Kakadiaris. Towards automatic reconstruction of dendrite morphology from live neurons. In Proc. $26^{\text {th }}$ Annual International Conference of the IEEE Engineering in Medicine and Biology Society, pages 1798-1801, San Fransisco, CA, Sep. 1-5 2004.

[73] Z. Vasilkoski and A. Stepanyants. Detection of the optimal neuron traces in confocal microscopy images. Journal of neuroscience methods, 178(1):197-204, 2009.

[74] Y. Wang, A. Narayanaswamy, C-L. Tsai, and B Roysam. A broadly applicable 3-D neuron tracing method based on open-curve snake. Neuroinformatics, 9(2-3):193 - 217, September 2011.

[75] S.L. Wearne, A. Rodriguez, D.B. Ehlenberger, A.B. Rocher, S.C. Henderson, and P.R. Hof. New techniques for imaging, digitization and analysis of three-dimensional neural morphology on multiple scales. Neuroscience, 136(3):661-680, 2005. Quantitative Neuroanatomy: from molecules to system. A special issue in honor of the late Professor Theodor W. Blackstad.

[76] Christina M. Weaver, Patrick R. Hof, Susan L. Wearne, and W. Brent Lindquist. Automated algorithms for multiscale morphometry of neuronal dendrites. Neural Computation, 16(7):1353-1383, Jul 2004.

[77] R. Yuste. Dendritic spines. MIT Press, 2009.

[78] R. Yuste and W. Denk. Dendritic spines as basic functional units of neuronal integration. Nature, 375(6533):682-684, June 1995.

[79] Rafael Yuste and Tobias Bonhoeffer. Genesis of dendritic spines: insights from ultrastructural and imaging studies. Nat Rev Neurosci, 5(1):24-34, Jan 2004.

[80] Y. Zhang, K. Chen, M. Baron, M.A. Teylan, Y. Kim, Z. Song, P. Greengard, and S.T.C. Wong. A neurocomputational method for fully automated $3 \mathrm{~d}$ dendritic spine detection and segmentation of medium-sized spiny neurons. NeuroImage, 50(4):1472-1484, May 2010.

[81] Y. Zhang, X. Zhou, R.M. Witt, B.L. Sabatini, D. Adjeroh, and S.T.C. Wong. Dendritic spine detection using curvilinear structure detector and LDA classifier. Neuroimage, 36(2):346-360, 2007. 